\title{
Effects of menstrual blood-derived stem cells on endometrial injury repair
}

\author{
$\mathrm{JIA} \mathrm{HU}^{1,2}$, KUANGYU SONG ${ }^{3}$, JING ZHANG ${ }^{2}$, YIQIONG ZHANG ${ }^{2}$ and BU-ZHEN TAN ${ }^{1}$ \\ ${ }^{1}$ Department of Obstetrics and Gynecology, The Second Affiliated Hospital of Nanchang University; \\ ${ }^{2}$ Department of Obstetrics and Gynecology, The First Affiliated Hospital of Nanchang University; \\ ${ }^{3}$ Department of Microbiology, School of Medicine, Nanchang University, Nanchang, Jiangxi 330006, P.R. China
}

Received October 16, 2017; Accepted August 15, 2018

DOI: $10.3892 / \mathrm{mmr} .2018 .9744$

\begin{abstract}
The present study aimed to investigate the effects of menstrual blood-derived stem cells (MenSCs) on endometrial injury repair. MenSCs were isolated from human menstrual blood and were cultured in vitro. Flow cytometric analysis of cells in the third generation demonstrated that MenSCs exhibited higher expression levels of cluster of differentiation (CD)90 and lower expression levels of CD146, which suggested that the MenSCs were cultured successfully. A mechanical damage model of unilateral (right) endometrium was established in BALB/c nude mice, which were divided into four groups, Normal, negative control (NC), Model and MenSC. MenSCs transfected with adenovirus-enhanced green fluorescent protein were transplanted into the right uterine cavity of mice in the MenSC and NC groups. The protein expression levels of keratin, vimentin, and vascular endothelial growth factor (VEGF) and the average endometrial thickness were measured by immunohistochemistry; the average optical density of vimentin, VEGF and keratin in the MenSC-treated group was significantly higher compared with the untreated Model group. Fertility tests were performed to determine the pregnancy rate of each group; following endometrial damage in BALB/c nude mice, endometrial thickness was decreased in the Model group, whereas model mice treated with MenSC exhibited increased endometrial thickness and increased the pregnancy rates. Therefore, MenSCs may promote the repair of endometrial lesions in mice by promoting the expression of vimentin, VEGF and keratin.
\end{abstract}

Correspondence to: Dr Bu-Zhen Tan, Department of Obstetrics and Gynecology, The Second Affiliated Hospital of Nanchang University, 1 Minde Road, Nanchang, Jiangxi 330006, P.R. China E-mail: tanbuzhen78@163.com

Key words: menstrual blood-derived stem cells, endometrial stromal cells, endometrial injury, pregnancy rate, vimentin, vascular endothelial growth factor

\section{Introduction}

The human uterus is a fibromuscular organ comprising three layers: Endometrium (innermost mucosal layer), myometrium (middle layer) and perimetrium (outer layer) (1). The endometrium is a multi-layered, dynamic organ overlaying the myometrium that is receptive to blastocyst implantation (2). Pregnancy requires effective cooperation between a receptive uterus and a superior blastocyst (3). In cattle and humans, the endometrium can be easily infected with bacteria, which may result in severe uterine diseases (4). Trauma or infection may cause endometrial repair disorder if trauma or infection damages the basal lamina of endometrium (5). An endometrium that is too thin is one of the most common causes of infertility in the uterus, which may cause embryos not to grow in the endometrium (6). Treatment of a thin endometrium consists mainly of drug therapy, stimulation scrape therapy and intrauterine injection of granulocyte colony-stimulating factor $(7,8)$. Although these multiple approaches to treatment are available, the overall curative outcome is poor, making this condition in reproductive medicine challenging.

Stem cells are undifferentiated cell populations with self-renewal and multilineage differentiation potential (9). Currently, the stem cells used for treatment of a thin endometrium include mesenchymal stem cells, human embryonic stem cells, endometrial stem cells and umbilical cord mesenchymal stem cells (10-13). In addition, successful repair of the endometrium in pregnancy with stem cells has been reported previously (14). A recent study demonstrated that the basal layer of the endometrium has high proliferative, self-renewal and differentiation potential of stem cells (15). Endometrial stem cells have been demonstrated to be ideal seed cells for the treatment of infertility caused by endometrial damage (16). However, direct access to endometrial stem cells is a process that may incur great damage. At present, the majority of endometrial stem cells are derived from the endometrium in patients undergoing hysterectomy (17), which means the source is limited. One study demonstrated that cast-off cells in endometrium isolated from menstrual blood may be cultured in vitro into becoming endometrial mesenchymal stem cells, and using this method, cells may proliferate at an increased rate (18). At the same time, because menstrual blood is considered human waste, investigators were able to avoid the problem 
of sensitive medical ethical issues. The use of menstrual blood-derived stem cell (MenSC) transplantation for repair of the endometrial injury in nude mice with endometrial injury has been previously investigated (19).

In the present study, density gradient centrifugation was used to isolate MenSCs. In addition, a model of endometrial injury in nude mice was established for subsequent exploration of the effects of MenSCs on the repair of endometrial injury. These data may provide a novel strategy for the clinical treatment of endometrial injuries in humans.

\section{Materials and methods}

Ethical statement. The present study was approved by the Ethics Committee of the First Affiliated Hospital of Nanchang University (Nanchang, China), and all participants provided written informed consent prior to enrolment in the study. Animal experiments and animal use were approved by the Ethical Committee of Animal experiments of the Second Affiliated Hospital of Nanchang University (Nanchang, China). The animal experiments followed published guidelines (20) for the conservation and application of laboratory animals; all efforts were made to minimize suffering.

Culture and identification of MenSCs. Between March 2016 and March 2017, menstrual blood was collected from 10 healthy women volunteers (age, 20-35 years). The volunteers had regular menstruation, with a menstrual cycle of 24-35 days (average, 28 days) and no medical complications. Cells extracted from menstrual blood were placed in PBS. Briefly, mononuclear cells of menstrual blood were separated by the method of density gradient centrifugation at $400 \mathrm{xg}$ for $15 \mathrm{~min}$ at room temperature with lymphocyte stratified liquid (GE Healthcare Life Sciences, Little Chalfont, UK) (21). Cells from the middle layer were extracted, washed with PBS, and the supernatant was then removed. Subsequently, cells were obtained via centrifugation at $400 \mathrm{x} g$ at room temperature for 5 min and then resuspended in Dulbecco's modified Eagle's medium (DMEM)/Ham's F-12 (cat. no. 12634; Gibco; Thermo Fisher Scientific, Inc., Waltham, MA, USA) supplemented with $10 \%$ fetal bovine serum (Gibco; Thermo Fisher Scientific, Inc.), mixed evenly, counted and adjusted to an appropriate density $\left(1 \times 10^{6}\right.$ cells $\left./ \mathrm{ml}\right)$ for cell culture. The cells were inoculated into DMEM high-glucose medium (Hyclone; GE Healthcare Life Sciences, Logan, UT, USA) containing 20\% fetal bovine serum (Gibco; Thermo Fisher Scientific, Inc.), $1 \%$ amphotericin B and 1\% mycillin (Gibco; Thermo Fisher Scientific, Inc.) for incubation. Following culturing for $72 \mathrm{~h}$ at $37^{\circ} \mathrm{C}$, the culture medium was changed every 3 days. When cell confluence reached $90 \%$, cell passage was conducted. Cells at the third generation were selected, re-suspended in PBS and placed into a $2 \mathrm{ml}$ Eppendorf tube $\left(2 \times 10^{5}\right.$ cells).

Cells were placed in an ice bath, fluorescent labelledassociated antibodies $(2 \mu \mathrm{leach})$ phycoerythrin-cyanine 5-conjugated cluster of differentiation (CD)90 (CD90-PE/Cy5; 1:1,000; cat. no. ab25272; Abcam, Cambridge, MA, USA) and fluorescein isothiocyanate (FITC)-conjugated CD146 (CD146-FITC; 1:1,000; cat. no. ab78451; Abcam, Cambridge, MA, USA) were added to each tube and incubated at $4^{\circ} \mathrm{C}$ for 1 h. Subsequently, cells were resuspended in PBS $(20 \mu 1)$.
Flow cytometry and FlowJo software version 6.1 (Tree Star, Inc., Ashland, OR, USA) was used to detect the expression of surface markers to identify MenSCs.

Adenovirus-enhanced green fluorescent protein (Ad-eGFP) transfection. Isolated MenSCs $\left(1 \times 10^{7}\right.$ cells $\left./ \mathrm{ml}\right)$ were seeded into a six-well plate, and Ad-eGFP $\left[2 \times 10^{8}\right.$ particle-forming units (PFU); final concentration, $\left.1 \times 10^{8} \mathrm{PFU} / \mathrm{ml}\right]$ was added to each well. Following transfection for $24 \mathrm{~h}$, MenSCs were observed under a fluorescence microscope. The MenSCs were digested by trypsin, and the transfection rate was detected by flow cytometry using FlowJo software version 6.1 (Tree Star, Inc.). Briefly, MenSCs were digested by $2.5 \mathrm{ml} / 1$ trypsin, centrifuged at $1,000 \mathrm{x} \mathrm{g}$ at room temperature for $3 \mathrm{~min}$ and then collected into a $1.5 \mathrm{ml}$ Eppendorf tube. Following this, DMEM/F12 medium (Gibco; Thermo Fisher Scientific, Inc.) was added to the tube to reach a volume of $200 \mu 1$, and cell suspension density was adjusted to $1 \times 10^{7}$ cells $/ \mathrm{ml}$. Following MenSC transplantation into the uterine cavity of model mice, levels of green fluorescence were determined with a BX53 fluorescence microscope (Olympus Corporation, Tokyo, Japan).

Establishment of a mechanical damage model of unilateral endometrium in BALB/c nude mice. A total of $109 \mathrm{BALB} / \mathrm{c}$ female nude mice (age, 10-12 weeks; weight, 17.5-20.5 g) purchased from Hunan SJA Laboratory Animal Co., Ltd. (Changsha, China) were used in this study, of which 79 mice were randomly selected and anaesthetized by intraperitoneal injection of $1 \%$ pentobarbital sodium $(0.75 \mathrm{ml} / 10 \mathrm{~g})$. Mice were housed at a temperature of $22-24^{\circ} \mathrm{C}$, a humidity of $45-70 \%$, a $12 / 12 \mathrm{~h} \mathrm{light/dark} \mathrm{cycle,} \mathrm{and} \mathrm{with} \mathrm{free} \mathrm{access}$ to food and water. When the mice had no limb reaction, they were placed in a supine position with the limbs fixed on the operating table with aseptic pads. The operation area was disinfected by $75 \%$ alcohol, and a longitudinal incision of $1.5 \mathrm{~cm}$ was made at the top of the bladder in the midline abdomen. Ophthalmic forceps were used to lift the skin, and ophthalmic scissors were used to cut the skin layer-by-layer longitudinally. In the back of the bladder, the bilateral uterus was touched, straight forceps were used to clamp the fallopian tubes with the cornu uteri (bilateral uterine horns) exposed. An incision was made in the right uterine horn, and a 4-gauge needle was inserted at a location $2 / 3$ of the distance between the cornu uteri and the corpus uterus and rotated to scratch the area 4 times. Subsequently, streptomycin $(100 \mathrm{mg} / \mathrm{ml})$ was used at the incision, and the muscles and skin were sutured layer-by-layer. Mechanical damage of the endometrium was established in the right uterus (Model group), whereas the left uterus was not treated (Normal group). Following suture, nursing care at $37^{\circ} \mathrm{C}$ was conducted to ensure recovery within $2 \mathrm{~h}$. Following rearing, 3 mice were sacrificed by cervical dislocation at days 1,3 and 5 , and the bilateral uterine horns exposed. The cornu uteri were fixed in $4 \%$ paraformaldehyde for $>24 \mathrm{~h}$, embedded in paraffin, and sectioned to a thickness of $4 \mu \mathrm{m}$. The sections were stained with hematoxylin and eosin (H\&E), as described below, and light microscopy was used to observe the conditions of the mechanically damaged endometrium model. 
Green fluorescence detection following MenSC transplantation into the uterine cavity of Model mice. From the 70 remaining model mice, 10 mice with specific-pathogen-free conditions were used in the present study. The MenSC cell suspension $\left(1 \times 10^{7}\right.$ cells $\left./ \mathrm{ml} ; 20 \mu \mathrm{l}\right)$ was injected into the right uterine cavity of 10 mechanically damaged Model mice, and the left uterine cavity was injected with equivalent PBS for use as the control. A total of 5 days post-injection, the mice were sacrificed, the abdominal cavity was opened and the uterine horns collected. Subsequently, the uterine horns were incubated with $4 \%$ paraformaldehyde for $24 \mathrm{~h}$ at room temperature and then dehydrated overnight in $20 \%$ sucrose at $4^{\circ} \mathrm{C}$. Following this, tissues were embedded in optimal cutting temperature compound (Sakura Finetek USA, Inc., Torrance, CA, USA) for $10-15 \mathrm{~min}$ at $-20^{\circ} \mathrm{C}$ and then sliced using a frozen section preparation instrument Cryostat (Leica Microsystems, Inc., Buffalo Grove, IL, USA) to establish frozen sections $(10 \mu \mathrm{m})$. Sections were then examined under a fluorescent electron microscope (magnification, x100).

Animal grouping. From the 60 remaining model mice, 30 mice were randomly selected and injected with a $20 \mu 1$ MenSC suspension $\left(1 \times 10^{7}\right.$ cells $\left./ \mathrm{ml}\right)$ into the damaged right uterine cavity (MenSC group), and 30 model mice were injected with equivalent PBS into the right uterine cavity (Model group). In addition, another 30 mice with undamaged right uterine horns were injected with $20 \mu \mathrm{l}$ MenSCs suspension into the right uterine cavity [negative control (NC) group]. The untreated left uteri of all groups was not injected with MenSCs or PBS and used as the Normal group ( $n=90 ; n=30$ per group). Following treatment, three mice from each group were sacrificed at days 1, 3 and 5. The uterus was dissected and bilateral uterine tissue was resected. The tissues were dehydrated in an increasing gradient of alcohol, cleared in xylene, embedded in paraffin wax, sectioned $(4 \mu \mathrm{m})$ and prepared for follow-up H\&E staining, as described below. At day 7 post-treatment, 21 mice in each group were randomly selected for fertility testing: Female mice (3 oestrus cycles; aged 11-13 weeks old; weight, 18.2-29.5 g) were placed in a cage with the same strain male mice (one female and male mouse per cage housed under the aforementioned conditions), which were purchased from Hunan SJA Laboratory Animal Co., Ltd (Changsha, China) and the pregnancy rate of mice in each group was observed.

$H \& E$ staining. H\&E-stained sections were used to observe the level of damage to the endometrium. Paraffin wax in the sections was removed by xylene and sections were washed with high to low concentrations of alcohol (100 alcohol, 95 alcohol, 80 alcohol and $70 \%$ alcohol) and distilled water for $3 \mathrm{~min}$, stained in haematoxylin for $3 \mathrm{~min}$, washed in running water 3 times, dehydrated with alcohol for $10 \mathrm{~min}$, stained with eosin, cleaned with xylene and mounted with resinene (Sigma-Aldrich; Merck KGaA, Darmstadt, Germany). Subsequently, the sections were observed under a light microscope (Olympus Corporation, Tokyo, Japan) and five different visual fields were photographed and examined per slide.

Immunohistochemistry (IHC) and average endometrial thickness measurement. Five days following grouping, sections were selected, dewaxed and rehydrated according to the aforementioned protocol and each slice was then supplemented with $50 \mu 1$ peroxidase blocking solution (Sigma-Aldrich; Merck KGaA) and incubated for $10 \mathrm{~min}$ at room temperature to block endogenous peroxidase activity. Following rinsing with PBS, the PBS solution was removed and the endometrial sections were incubated with $50 \mu \mathrm{l}$ of normal non-immune animal serum (Sigma-Aldrich; Merck $\mathrm{KGaA}$ ) for $10 \mathrm{~min}$ at room temperature to remove serum. Subsequently, primary antibodies against vimentin (1:200; cat. no. ab92547; Abcam, Cambridge, UK), vascular endothelial growth factor (VEGF; 1:100; cat. no. ab9546; Abcam) and keratin (1:200; cat. no. ab8068; Abcam) were added to the sections and incubated at $4^{\circ} \mathrm{C}$ overnight. The sections were placed at room temperature for $1 \mathrm{~h}$, washed three times with PBS, 10 min each. Sheep anti-rabbit IgG antibodies (1:1,000; cat. no. ab6795; Abcam) were added to the sections and incubated at room temperature for $2 \mathrm{~h}$. Following treatment with 3,3'-Diaminobenzidine at $37^{\circ} \mathrm{C}$ for $5 \mathrm{~min}$, the sections were washed in PBS and counterstained with 5\% hematoxylin solution (Beyotime Institute of Biotechnology, Haimen, China) for 3 min at room temperature. Following development, sections were photographed using a light microscope (magnification, x400; Olympus Corporation), and five different visual fields were analysed. Image-Pro Plus 6 processing software (Media Cybernetics, Inc., Rockville, MD, USA) was used to analyse results and to calculate average optical density (AOD); $\mathrm{AOD}=$ integrated $\mathrm{OD} / \mathrm{measurement}$ area. The higher the AOD is, the higher the expression of the protein. Magnification at $x 400$ was also used to measure the average endometrial thickness.

Statistical analysis. Statistical analysis was conducted with SPSS 21.0 (IBM Corp., Armonk, NY, USA). Enumeration data were presented by percentage and tested by $\chi^{2}$, whereas measurement data were presented as the mean \pm standard deviation. Comparisons between two groups were conducted by unpaired Student's t-test, and comparisons among multiple groups were analysed using one-way analysis of variance followed by Bonferroni correction. $\mathrm{P}<0.05$ was considered to indicate a statistically significant difference.

\section{Results}

Cultured menstrual blood cells have stem cell characteristics. Following the culture of MenSCs for $24 \mathrm{~h}$, tadpole and polygonal adherent cells were observed under a microscope (Fig. 1A). Following 72 h, the whole medium was replaced, and impure cells were removed. Following culturing for 3-4 days, cells were not of uniform size and presented as a colony growth with uneven distribution. Following culturing for seven days, a small number of polygonal cells were observed, and most cells were clearly fusiform with rich and transparent cytoplasm and oval and cantered nuclei; in addition, cells grew in adherence to a non-polar arrangement (Fig. 1A). Flow cytometric analysis of cells at the third generation demonstrated that CD90-PE-positive cells accounted for 96.2\% and CD146-FITC-positive cells accounted for $0.014 \%$ (Fig. 1B and C, respectively), which indicated a high expression of the haematopoietic stem cell marker CD90 and low expression of mesenchymal stem cell marker CD146. These 
A

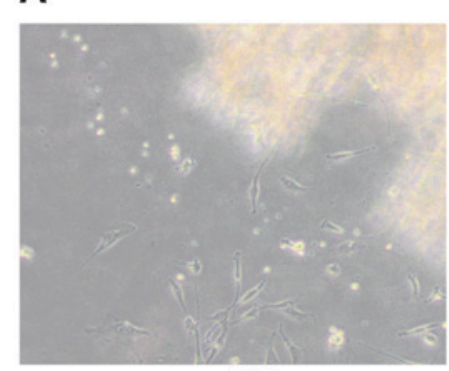

$24 \mathrm{~h}$

B

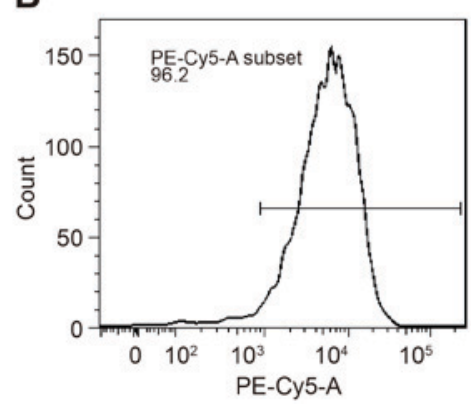

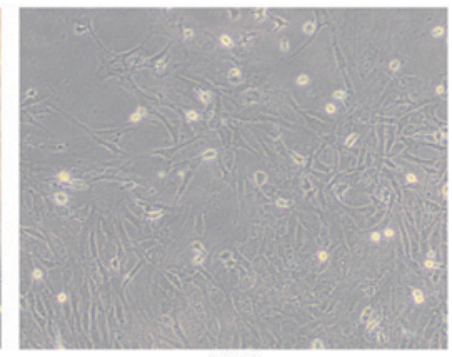

$72 \mathrm{~h}$

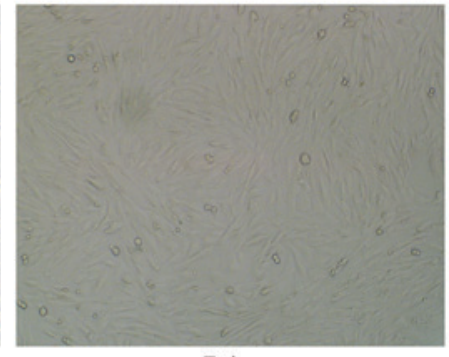

$7 d$

C

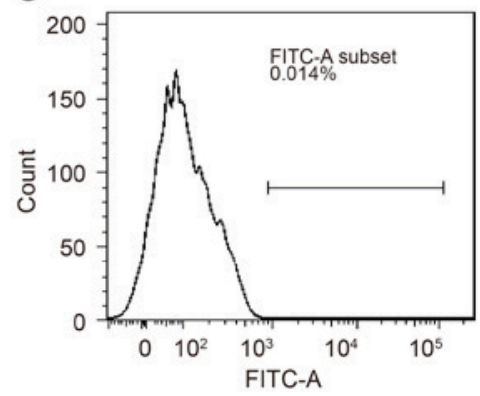

Figure 1. Culture and identification of MenSCs. (A) Morphological observation of MenSCs at different time points: Left panel, cell morphology at the $24 \mathrm{~h}$ time interval (magnification, x100); middle panel, cell morphology at the $72 \mathrm{~h}$ time interval (magnification, x100); right panel, cell morphology at the 7 day time interval (magnification, x100). (B and C) MenSCs examined by flow cytometry exhibited (B) high CD90-PE expression and (C) low CD146-FITC expression. $\mathrm{CD}$, cluster of differentiation; FITC, fluorescein isothiocyanate; MenSCs, menstrual blood-derived stem cells; PE, phycoerythrin.

results demonstrated that the cultured cells exhibited stem cell characteristics.

Successful establishment of a mechanically damaged endometrium model in mice. The unilateral injury model of nude mice was established via mechanical injury, and following modelling for 1,3 , and 5 days, the uteri were collected and stained with H\&E. Detached endometrial tissue was observed in the Model group at the 1 day time interval, fibrosis was observed in the endometrium of the Model group at the 3 day time interval, and no normal endometrium was observed while fibrosis was observed in the endometrium of the Model group at the 5 day time interval; whereas no fibrosis was detected in the Normal group (Fig. 2). These results indicated that the endometrial mechanical damage model was successfully established.

MenSCs grow in the endometrium of model mice. Following transfection for $24 \mathrm{~h}$, Ad-eGFP-MenSCs were observed under a fluorescence microscope, and the transfection rate was up to 48.5\% (Fig. 3A and B). Ad-eGFP-MenSCs were transplanted into the right uterus of model mice (MenSC group), and PBS was injected into the Model group mice; in the MenSC-treated model mice, green fluorescence was detected in the endometrium (Fig. 3C), which suggested that the MenSCs were able to grow in the endometrium of nude mice.

MenSC treatment increases endometrial thickness and promotes endometrial repair. At 5 days post-treatment, the results of IHC demonstrated that the endometrial thickness of the Model group was thinner compared with the Normal group $(\mathrm{P}<0.05$; Fig. 4); the endometrial thickness of mice in the MenSC group was increased compared with mice in the untreated Model group, but no significant difference was identified $(\mathrm{P}>0.05)$. In addition, the endometrial thickness of the MenSC group was significantly decreased compared with normal group $(\mathrm{P}<0.05$; Fig. 4). Compared with the NC group, the endometrial thickness of Model and MenSC groups were significantly decreased $(\mathrm{P}<0.05$; Fig. 4). No significant difference was detected in a comparison of the endometrial thickness between the Normal and NC groups ( $\mathrm{P}>0.05$; Fig. 4). These results revealed that endometrial thickness decreased following endometrial damage, and this effect may be reversed following MenSC treatment in model mice.

MenSCs promote the repair of endometrium by promoting the expression of vimentin, VEGF and keratin. Keratin is expressed in epithelial cells, which are associated with epithelial growth (22). Vimentin is mainly expressed in mesenchymal cells and endothelial cells, which are associated with stromal cell regrowth (22). VEGF is associated with angiogenesis (23). Thus, keratin, vimentin and VEGF are associated with endometrial repair. At 5 days post-treatment, the results of IHC demonstrated that positive staining of vimentin, VEGF and keratin was brown or tan (Fig. 5). No significant difference was detected in the AOD of vimentin, VEGF and keratin between the Normal and NC groups $(\mathrm{P}>0.05)$. The AOD of vimentin, VEGF and keratin in the Model group was significantly lower compared with the respective expression levels in the Normal and $\mathrm{NC}$ groups $(\mathrm{P}<0.05)$. The AOD of vimentin, VEGF and keratin in the MenSC group were significantly higher compared with the respective expression levels in the untreated Model group $(\mathrm{P}<0.05)$. These data indicated that MenSCs may promote the repair of endometrial lesions in model mice by promoting the expression of vimentin, VEGF and keratin. 


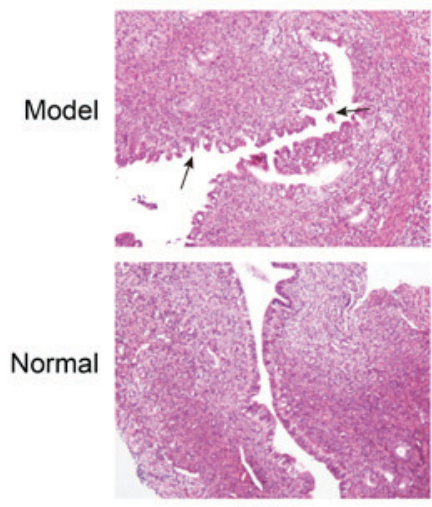

Day 1

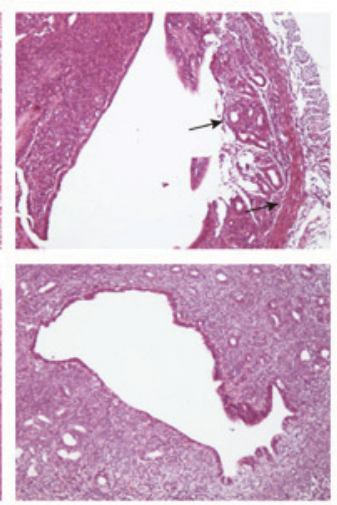

Day 3

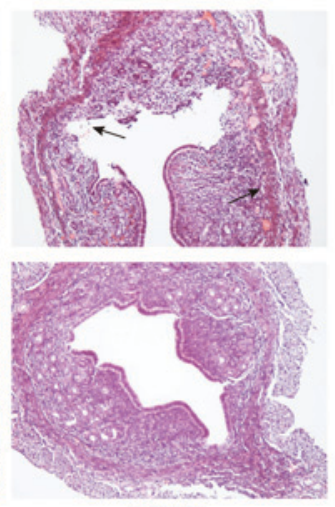

Day 5

Figure 2. Examination of endometrium in Model mice. Right uterine horns were collected from Model mice and examined for fibrosis by haematoxylin and eosin staining at days 1,3 and 5 following endometrial damage; the left uterine horns were undamaged and used as a Normal control. Black arrow indicates endometrial fibrosis. Magnification, x100.
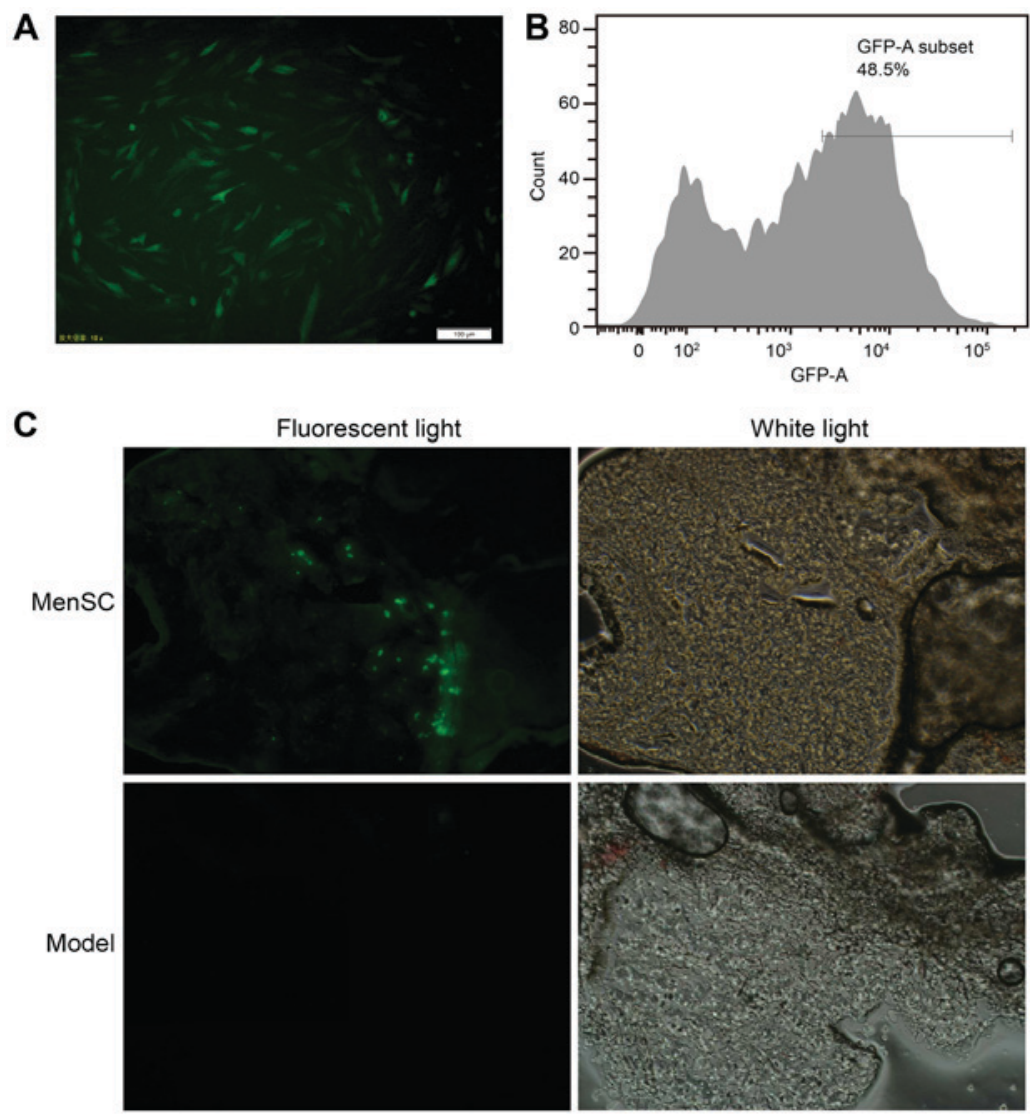

Figure 3. Ad-eGFP-transfected MenSCs are able to grow in nude mice. (A and B) MenSCs were transfected with Ad-eGFP and (A) observed under a fluorescence microscope and (B) the number of positive (green) cells were quantified by flow cytometry at $24 \mathrm{~h}$ following transfection. (C) Ad-eGFP-transfected MenSCs were transplanted into the right uterine horn of endometrial damaged model mice and endometrial sections were observed under a fluorescence microscope; Model control mice received endometrial damage and treated with PBS. Ad-eGFP, adenovirus-enhanced green fluorescent protein; MenSCs, menstrual blood-derived stem cells.

MenSCs increase the fertility of damaged endometrium in nude mice. Fertility tests demonstrated that the undamaged left uterus in mice from each group were successfully impregnated (42 out of 42; 100\%; Table I). In the Model group, 4 pregnancies occurred out of 21 mice with right uterine horn damage (19.04\%), whereas 11 out of 21 pregnancies occurred in the MenSC-treated group (52.38\%), which was significantly higher compared with the untreated Model group $(\mathrm{P}<0.05)$. The results demonstrated that MenSC treatment may improve the fertility rates in Model mice with the damaged endometrium.

\section{Discussion}

Good endometrial receptivity, which is indicated by endometrial thickness and blood flow, is an important condition for a successful pregnancy $(24,25)$. Previous studies have demonstrated that the 

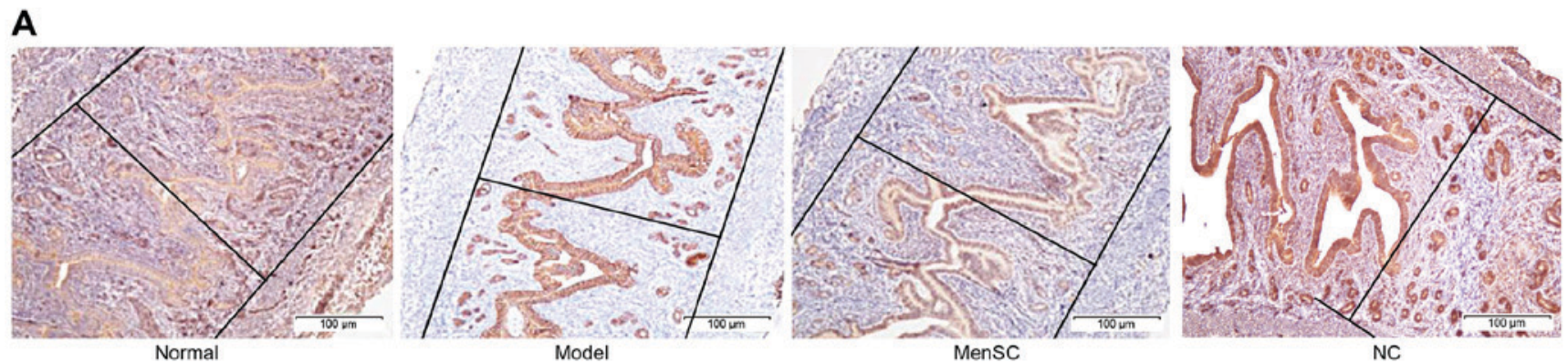

B

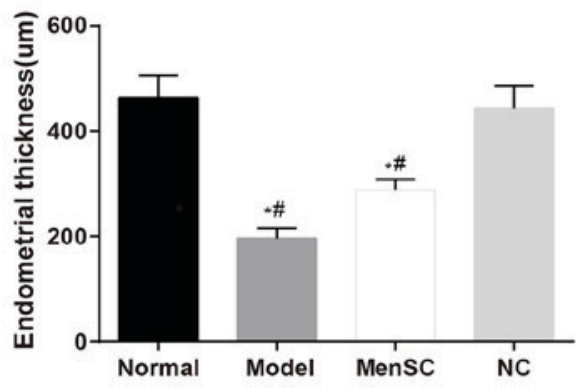

Figure 4. MenSCs treatment affects the thickness of the damaged endometrium in Model mice. (A) Immunohistochemical staining of the endometrium from mice from each group; magnification, $\mathrm{x} 40$. (B) Quantification of the endometrial thickness in mice from each group. ${ }^{\text {}} \mathrm{P}<0.05$ vs. Normal. ${ }^{\#} \mathrm{P}<0.05$ vs. NC. MenSCs, menstrual blood-derived stem cells; NC, negative control.

A
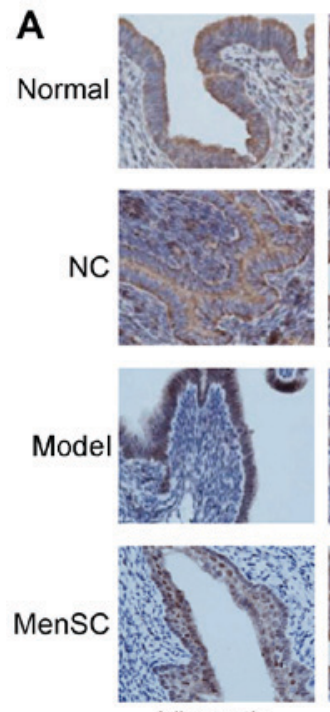

Vimentin
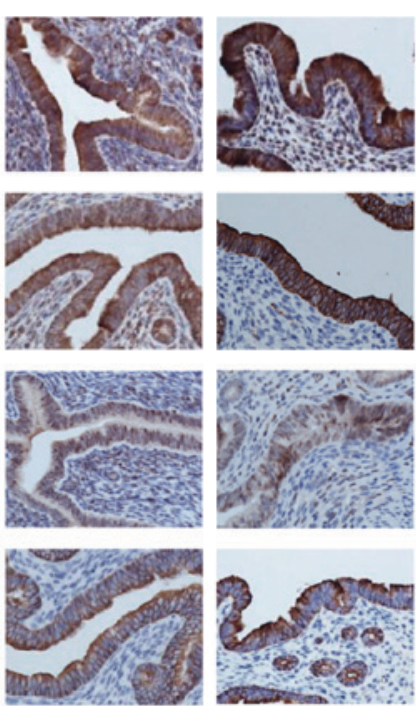

Keratin

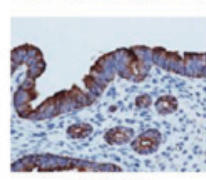

B

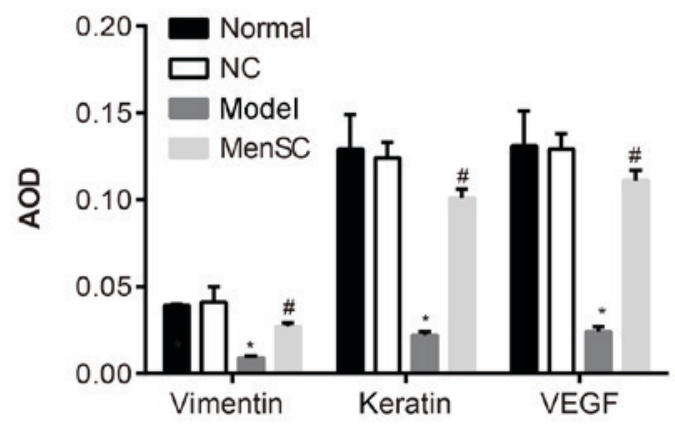

Figure 5. Expression of vimentin, VEGF and keratin proteins in the endometrium of Model mice. (A) Immunohistochemical staining of endometrium from mice in each group; magnification, $\mathrm{x} 400$. (B) Quantification of the expression of vimentin, VEGF and keratin from Part A. "P<0.05 vs. Normal; "P $<0.05$ vs. Model. AOD, average optical density; MenSCs, menstrual blood-derived stem cells; NC, negative control; VEGF, vascular endothelial growth factor.

pregnancy rates of patients with endometrial thickness $<7 \mathrm{~mm}$ was significantly decreased $(24,25)$. Although there are several treatment strategies for a thin endometrium currently available, some patients receiving treatment still have a low response or none at all $(26,27)$. The present study demonstrated that MenSCs could be isolated from menstrual blood and used to promote the repair of endometrial lesions in nude mice.

A previous study reported that bone marrow mesenchymal stem cell transplantation could be used for the treatment of intrauterine adhesion syndrome (28). Another study demonstrated that MenSCs were able to differentiate into endometrial epithelial cells (29). Previous studies have also demonstrated that MenSCs exhibited positive expression of the haematopoietic stem cell marker CD90 and negative expression of the mesenchymal stem cell marker CD146 $(30,31)$. Similarly, in the present study, MenSCs were isolated from healthy women and cultured in vitro; these cells exhibited stem cell-like characteristics with high CD90 expression and low CD46 expression. At present, mechanical and thermal injury are commonly used methods for establishing a model of endometrial injury $(28,32)$. However, these models used normal immune mice, and the establishment of similar nude mouse models have not yet been 
Table I. Pregnancy rates of mice in each group.

\begin{tabular}{lcccc}
\hline Fertility status & Normal $(\mathrm{n}=21)^{\mathrm{a}}$ & NC $(\mathrm{n}=21)^{\mathrm{b}}$ & Model $(\mathrm{n}=21)^{\mathrm{b}}$ & ${\text { MenSC }(\mathrm{n}=21)^{\mathrm{b}}}^{\mathrm{b}}$ \\
\hline Pregnancy & 21 & 21 & 4 & 11 \\
Not pregnancy & 0 & 0 & 17 & 10 \\
Pregnancy rate $(\%)$ & 100 & 100 & 19.04 & $52.38^{\mathrm{c}}$ \\
\hline
\end{tabular}

${ }^{a}$ Normal group comprised the left uterine horns from the Control and MenSC group. ${ }^{\text {bRight }}$ uterine horn only. ${ }^{\mathrm{c}} \mathrm{P}<0.05 \mathrm{vs}$. Model. MenSC, menstrual blood-derived stem cell; $\mathrm{NC}$, negative control.

reported. In the present study, a model of endometrial injury in nude mice was successfully constructed using the mechanical damage method by Alawadhi et al (28).

Thin endometrium is characterized by slow growth of the glandular epithelium; therefore, the regeneration of epithelial cells is especially important for the improvement of the thin endometrium (33). MenSCs transfected with Ad-eGFP were able to grow in the endometrium of nude mice. It was demonstrated that endometrial thickness decreased following endometrial damage, while MenSCs treatment increased endometrial thickness, promoted endometrial repair and increased the fertility of damaged endometrium in nude mice. These results were similar to a previous study, which demonstrated that MenSCs were able to repair injured endometrium and increase the fertility of mice suffering endometrial injury (34). Furthermore, the present study demonstrated that MenSC-treated model mice exhibited increased protein expression levels of vimentin, VEGF and keratin. Vimentin is a cytoskeletal protein that is mainly expressed in the cytoskeleton of mesenchymal cells and functions to maintain the stability of cell structure and is involved in mitosis, differentiation, proliferation and signal transduction (35). VEGF is a highly specific mitogen of vascular endothelial cells and is an important regulator of angiogenesis; its expression reflects the level of proliferation, migration and vascular construction of vascular endothelial cells $(36,37)$. VEGF is expressed in the glandular epithelium and vascular endothelial cells of the thin and normal endometrium, and the expression of VEGF in the thin endometrium was lower (38). Another study demonstrated that icariin treatment increase the thin endometrium in a rat model of intimal thickness by upregulation of VEGF expression (39). Keratin is a protein marker of epithelial cells, which serves an important role in maintaining the integrity of epithelial cells (40). A previous study demonstrated increased expression of keratin in endometrial tissues (41). In agreement with the results of the present study, Zhu et al (19) demonstrated that MenSC-based therapies may represent promising methods for the treatment of endometrial injury via the regulation of AKT and p38 signalling pathways.

In conclusion, the present study demonstrated that MenSCs could be isolated from the menstrual blood and were able to survive in the endometrium of nude mice with endometrial damage. Furthermore, MenSCs were able to improve the embryo implantation rate, possibly by promoting the expression levels of vimentin, keratin and VEGF. Additional experiments are required to explore the possible molecular mechanisms underlying the association between MenSCs and endometrial fibrosis.

\section{Acknowledgements}

The authors would like to acknowledge the critical revision of the manuscript by the reviewers.

\section{Funding}

The present study was supported by the Jiangxi Provincial Department of Health (grant no. 20155114).

\section{Availability of data and materials}

The analysed data sets generated during the present study are available from the corresponding author on reasonable request.

\section{Authors' contributions}

$\mathrm{JH}$ performed the animal experiments as well as wrote and revised the manuscript. BZT contributed to the experimental design. KYS performed cell culture, adenovirus transfection, green fluorescence detection and immunohistochemistry experiments. JZ collected the menstrual blood samples and cultured the menstrual blood-derived stem cells. YQZ collected and analysed the data.

\section{Ethics approval and consent to participate}

The present study was approved by the Ethics Committee of the First Affiliated Hospital of Nanchang University (Nanchang, China), and all participants provided written informed consent prior to enrolment in the study. Animal experiments and animal use were approved by the Ethical Committee of Animal experiments of the Second Affiliated Hospital of Nanchang University. The animal experiments followed the guidelines for the conservation and application of laboratory animals; all efforts were made to minimize suffering.

\section{Patient consent for publication}

The subjects provided informed consent and agreed to the publication of this article.

\section{Competing interests}

The authors declare that they have no competing interests. 


\section{References}

1. Fiocchi F, Nocetti L, Siopis E, Currà S, Costi T, Ligabue G and Torricelli P: In vivo 3 T MR diffusion tensor imaging for detection of the fibre architecture of the human uterus: A feasibility and quantitative study. Br J Radiol 85: e1009-e1017, 2012.

2. Maryam KS, Christopher MR, Ahmad H and Valojerdi RM: Ultrastructural modifications of human endometrium during the window of implantation. Int J Fertil Steril 2: 44-59, 2008.

3. Hyodo S, Matsubara K, Kameda K and Matsubara Y: Endometrial injury increases side population cells in the uterine endometrium: A decisive role of estrogen. Tohoku J Exp Med 224: 47-55, 2011.

4. Davies D, Meade KG, Herath S, Eckersall PD, Gonzalez D, White JO, Conlan RS, O'Farrelly C and Sheldon IM: Toll-like receptor and antimicrobial peptide expression in the bovine endometrium. Reprod Biol Endocrinol 6: 53, 2008.

5. Deans R and Abbott J: Review of intrauterine adhesions. J Minim Invasive Gynecol 17: 555-569, 2010.

6. Mahajan N and Sharma S: The endometrium in assisted reproductive technology: How thin is thin? J Hum Reprod Sci 9: 3-8, 2016.

7. Chen JR, Cheng JG, Shatzer T, Sewell L, Hernandez L and Stewart CL: Leukemia inhibitory factor can substitute for nidatory estrogen and is essential to inducing a receptive uterus for implantation but is not essential for subsequent embryogenesis. Endocrinology 141: 4365-4372, 2000.

8. Santjohanser C, Knieper C, Franz C, Hirv K, Meri O, Schleyer M, Würfel W and Toth B: Granulocyte-colony stimulating factor as treatment option in patients with recurrent miscarriage. Arch Immunol Ther Exp (Warsz) 61: 159-164, 2013.

9. Fossett E, Khan WS, Longo UG and Smitham PJ: Effect of age and gender on cell proliferation and cell surface characterization of synovial fat pad derived mesenchymal stem cells. J Orthop Res 30: 1013-1018, 2012.

10. Wang J, Ju B, Pan C, Gu Y, Zhang Y, Sun L, Zhang B and Zhang Y: Application of bone marrow-derived mesenchymal stem cells in the treatment of intrauterine adhesions in rats. Cell Physiol Biochem 39: 1553-1560, 2016.

11. Song T, Zhao X, Sun H, Li X, Lin N, Ding L, Dai J and Hu Y: Regeneration of uterine horns in rats using collagen scaffolds loaded with human embryonic stem cell-derived endometrium-like cells. Tissue Eng Part A 21: 353-361, 2015.

12. Darzi S, Werkmeister JA, Deane JA and Gargett CE: Identification and characterization of human endometrial mesenchymal stem/stromal cells and their potential for cellular therapy. Stem Cells Transl Med 5: 1127-1132, 2016.

13. Huang P, Lin LM, Wu XY, Tang QL, Feng XY, Lin GY, Lin X, Wang HW, Huang TH and Ma L: Differentiation of human umbilical cord Wharton's jelly-derived mesenchymal stem cells into germ-like cells in vitro. J Cell Biochem 109: 747-754, 2010.

14. Figueira PG, Abrão MS, Krikun G and Taylor HS: Stem cells in endometrium and their role in the pathogenesis of endometriosis. Ann N Y Acad Sci 1221: 10-17, 2011.

15. Xu S, Chan RWS, Ng EHY and Yeung WSB: Spatial and temporal characterization of endometrial mesenchymal stem-like cells activity during the menstrual cycle. Exp Cell Res 350: 184-189, 2017.

16. Phermthai T, Tungprasertpol K, Julavijitphong S, Pokathikorn P, Thongbopit S and Wichitwiengrat S: Successful derivation of xeno-free mesenchymal stem cell lines from endometrium of infertile women. Reprod Biol 16: 261-268, 2016.

17. Schwab KE and Gargett CE: Co-expression of two perivascular cell markers isolates mesenchymal stem-like cells from human endometrium. Hum Reprod 22: 2903-2911, 2007.

18. Musina RA, Belyavski AV, Tarusova OV, Solovyova EV and Sukhikh GT: Endometrial mesenchymal stem cells isolated from the menstrual blood. Bull Exp Biol Med 145: 539-543, 2008.

19. Zhu H, Jiang Y, Pan Y, Shi L and Zhang S: Human menstrual blood-derived stem cells promote the repair of impaired endometrial stromal cells by activating the p38 MAPK and AKT signaling pathways. Reprod Biol 18: 274-281, 2018.

20. Jones-Bolin S: Guidelines for the care and use of laboratory animals in biomedical research. Curr Protoc Pharmacol Appendix 4: Appendix 4B, 2012.

21. Liu T, Huang Y, Zhang J, Qin W, Chi H, Chen J, Yu Z and Chen C: Transplantation of human menstrual blood stem cells to treat premature ovarian failure in mouse model. Stem Cells Dev 23: 1548-1557, 2014.
22. Velez-delValle C, Marsch-Moreno M, Castro-Muñozledo F, Galván-Mendoza IJ and Kuri-Harcuch W: Epithelial cell migration requires the interaction between the vimentin and keratin intermediate filaments. Sci Rep 6: 24389, 2016.

23. Rosenbaum-Dekel Y, Fuchs A, Yakirevich E, Azriel A, Mazareb S, Resnick MB and Levi BZ: Nuclear localization of long-VEGF is associated with hypoxia and tumor angiogenesis. Biochem Biophys Res Commun 332: 271-278, 2005.

24. Chakrabarti J, Chatterjee R, Goswami S, Chakravarty B and Kabir SN: Overt leptin response to controlled ovarian hyperstimulation negatively correlates with pregnancy outcome in in vitro fertilization-embryo transfer cycle. J Hum Reprod Sci 5: 194-199, 2012.

25. Chien LW, Au HK, Chen PL, Xiao J and Tzeng CR: Assessment of uterine receptivity by the endometrial-subendometrial blood flow distribution pattern in women undergoing in vitro fertilization-embryo transfer. Fertil Steril 78: 245-251, 2002.

26. Aydin T, Kara M and Nurettin T: Relationship between endometrial thickness and in vitro fertilization-intracytoplasmic sperm injection outcome. Int J Fertil Steril 7: 29-34, 2013

27. Chen SL, Wu FR, Luo C, Chen X, Shi XY, Zheng HY and Ni YP: Combined analysis of endometrial thickness and pattern in predicting outcome of in vitro fertilization and embryo transfer: A retrospective cohort study. Reprod Biol Endocrinol 8: 30, 2010.

28. Alawadhi F, Du H, Cakmak H and Taylor HS: Bone Marrow-Derived Stem Cell (BMDSC) transplantation improves fertility in a murine model of Asherman's syndrome. PLoS One 9: e96662, 2014.

29. Jiang YS, Zhu HY, Jin XY, Li C, Li J, Zhang YL and Zhang SY: Co-culture with endometrial stromal cells promotes the differentiation of menstrual blood-derived mesenchymal stem cells into endometrial epithelial cells. Zhonghua Yi Xue Za Zhi 97: 2614-2619, 2017 (In Chinese).

30. Mehrabani D, Nazarabadi RB, Kasraeian M, Tamadon A, Dianatpour M, Vahdati A, Zare S and Ghobadi F: Growth kinetics, characterization, and plasticity of human menstrual blood stem cells. Iran J Med Sci 41: 132-139, 2016.

31. Tsuji S, Yoshimoto M, Takahashi K, Noda Y, Nakahata T and Heike T: Side population cells contribute to the genesis of human endometrium. Fertil Steril 90 (Suppl 4): S1528-S1537, 2008.

32. Hu X, Lin X, Jiang Y, Shi L, Wang J, Zhao L and Zhang S: Establishment of mouse endometrial injury model by electrocoagulation. Zhonghua Yi Xue Za Zhi 94: 3771-3773, 2014 (In Chinese).

33. Miwa I, Tamura H, Takasaki A, Yamagata Y, Shimamura K and Sugino N: Pathophysiologic features of 'thin' endometrium. Fertil Steril 91: 998-1004, 2009.

34. Zhang Y, Lin X, Dai Y, Hu X, Zhu H, Jiang Y and Zhang S: Endometrial stem cells repair injured endometrium and induce angiogenesis via AKT and ERK pathways. Reproduction 152: 389-402, 2016

35. Zhou J, Tao D, Xu Q, Gao Z and Tang D: Expression of E-cadherin and vimentin in oral squamous cell carcinoma. Int J Clin Exp Pathol 8: 3150-3154, 2015.

36. Yang F, Tang X, Riquelme E, Behrens C, Nilsson MB, Giri U, Varella-Garcia M, Byers LA, Lin HY, Wang J, et al: Increased VEGFR-2 gene copy is associated with chemoresistance and shorter survival in patients with non-small-cell lung carcinoma who receive adjuvant chemotherapy. Cancer Res 71: 5512-5521, 2011.

37. Torabinia N, Razavi SM and Tahririan D: Vascular Endothelial Growth Factor (VEGF) expression in normal, dysplastic and neoplastic squamous epithelium of oral mucosa. J Pioneer Med Sci 4: 115-118, 2014.

38. Vlahos NF, Gregoriou O, Deliveliotou A, Perrea D, Vlachos A, Zhao Y, Lai J and Creatsas G: Effect of pentoxifylline on vascular endothelial growth factor $\mathrm{C}$ and flk-1 expression on endometrial implants in the rat endometriosis model. Fertil Steril 93: 1316-1323, 2010.

39. Le AW, Wang ZH, Dai XY, Xiao TH, Zhuo R, Zhang BZ, Xiao ZL and Fang XJ: An experimental study on the use of icariin for improving thickness of thin endometrium. Genet Mol Res 16, 2017.

40. Karantza V: Keratins in health and cancer: More than mere epithelial cell markers. Oncogene 30: 127-138, 2011.

41. Ikoma T, Kyo S, Maida Y, Ozaki S, Takakura M, Nakao S and Inoue $\mathrm{M}$ : Bone marrow-derived cells from male donors can compose endometrial glands in female transplant recipients. Am J Obstet Gynecol 201: 608.e1-e8, 2009.

This work is licensed under a Creative Commons

Attribution-NonCommercial-NoDerivatives 4.0 International (CC BY-NC-ND 4.0) License. 